\title{
puente mixto para ferrocarril y carretera
}

P. C. HARRIS, ingeniero

$569-9$

\section{simopsis}

El nuevo puente, llamado West Branch, que salva el profundo cauce de un río, tiene una doble finalidad y dos tableros superpuestos: uno para carretera en la parte superior y'otro para una vía férrea. Ha sido realizado por el Departamento de Obras Pú. blicas de California. La estructura consta de cuatro tramos principales apoyados sobre pilas de hormigón armado, de gran altura y en cuyo interior se han dejado cuatro células o huecos de seccion rectangular. El conjunto de los dos tableros está constituido por un entramado metalico, continuo, montado siguiendo los procedimientos cantilever, es decir, en voladizo. La celosía, a base de elementos muy pesados, de hasta de cér la cota. El hormiconado de el hormigón inicial en larte duperior del encofrado tuviera cuatro horas de vejez al pasar la parte inferior del encofrado por el hor Para lo nivel. Para is del encofrado. La maniobra de este elevador del hormigón, cuya energía era suministrada por un torno instalado en el suelo, se dirigía, por comunicación telefónica, entre el operador del torno y el encargado de distribuir el hormigón en la plataforma superior de trabajo. Para la terminación y acabado de los paramentos que dejaba el encofrado al deslizar en elevación, se preparó una plataforma auxiliar de trabajo en la parte inferior del encofrado exterior y otro tanto se hizo para los encofrados interiores correspondientes a las cuatro células o huecos interiores de las pilas.

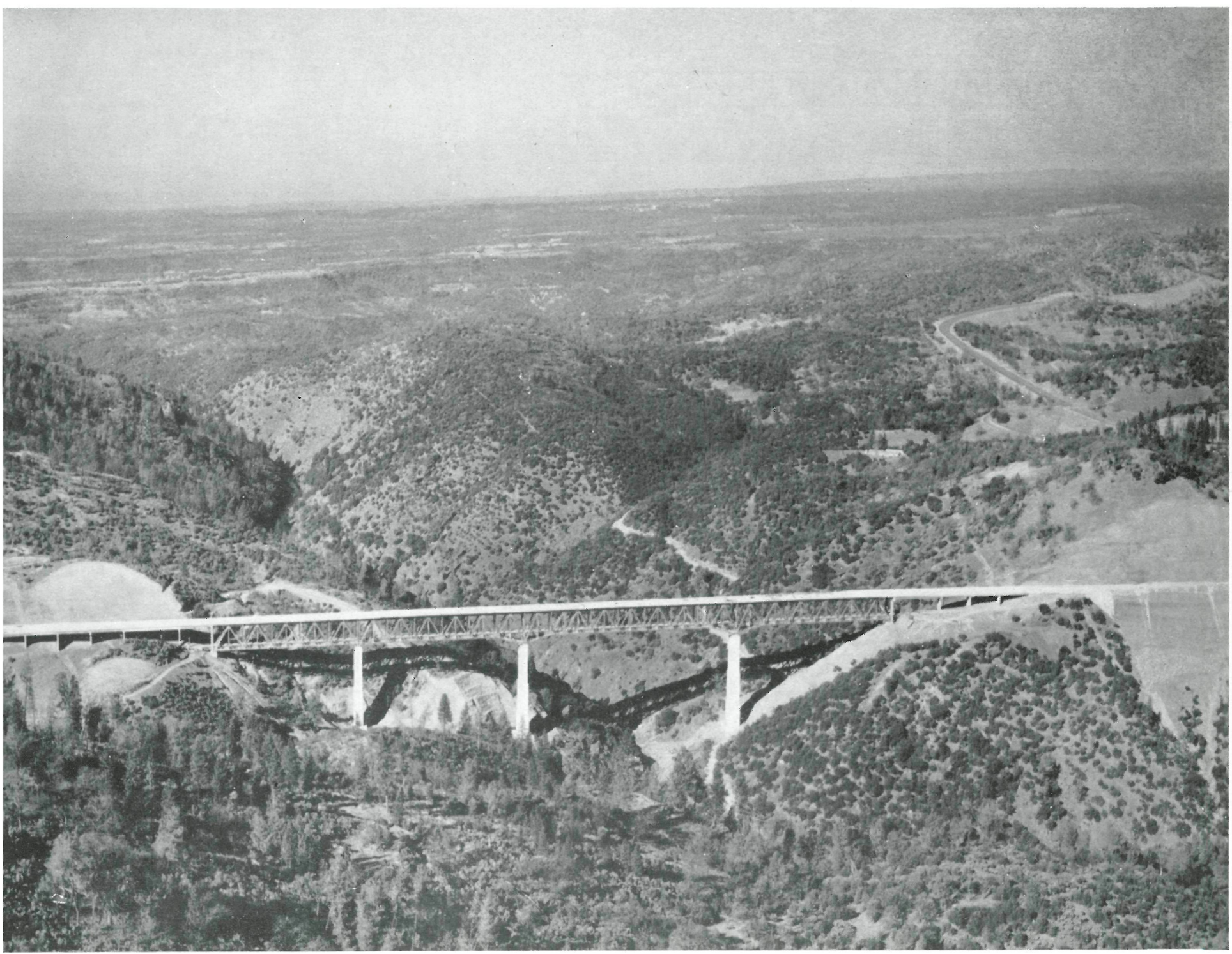


El nuevo puente para ferrocarril y carretera, denominado West Branch Bridge, se halla situado en la zona de Butte County, Distrito III de California (EE. UU.). La estructura, metálica, soporta una carretera de cuatro bandas de circulación en el tablero superior y una vía férrea simple en el inferior.

La parte principal de la estructura consta de cuatro tramos rectos de 109, 132, 175 y $132 \mathrm{~m}$ de luz, respectivamente, apoyados sobre pilas de hormigón de gran altura.

En una extremidad los accesos están constituidos, para la carretera, por cinco tramos de $24 \mathrm{~m}$ de luz cada uno y un tramo de $40 \mathrm{~m}$, mientras que en la otra extremidad sólo tiene tres tramos de $24 \mathrm{~m}$ y otro de también $40 \mathrm{~m}$. La longitud total para el tablero de carretera es de 832 metros, aproximadamente, y de 572 en la del tablero que soporta la vía férrea. Las cerchas o celo-

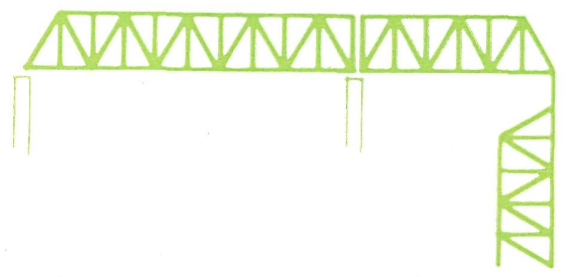
sías principales se han espaciado a $11,5 \mathrm{~m}$ y tie-
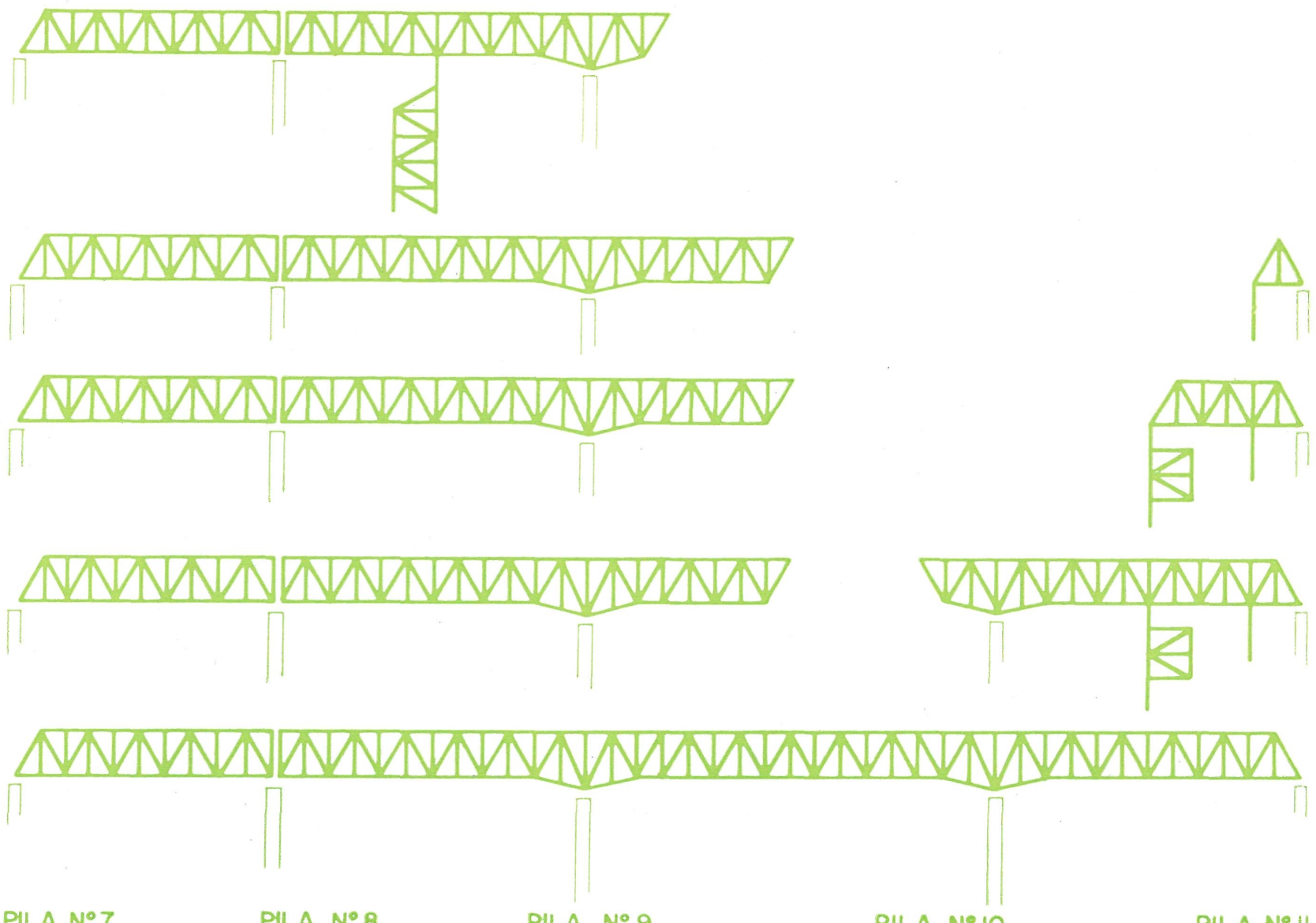

\section{esquema de montaje}


nen un canto de $18 \mathrm{~m}$, excepto sobre las dos pilas principales, que es de $24 \mathrm{~m}$. Dichas pilas, de hormigón, tienen una altura variable, con un máximo de $87 \mathrm{~m}$ entre cimiento y apoyo superior.

Los cimientos de la pila de mayor volumen tienen $5,5 \mathrm{~m}$ de profundidad y $25 \times 21 \mathrm{~m}$ en planta Sobre este cimiento se eleva una base de $21 \times 15$ metros en planta y $4,80 \mathrm{~m}$ de altura. El cuerpo superior se compone de otros tres bloques de hormigón: El inferior tiene $17,6 \times 7,30 \mathrm{~m}$ en planta y $17,6 \mathrm{~m}$ de altura; el inmediato superior, $17 \times 6$ metros en planta y $24 \mathrm{~m}$ de altura, y el tercero y último, $16,40 \times 4,80 \mathrm{~m}$ en planta y $30 \mathrm{~m}$ de altura. En el interior de estas pilas se han formado cuatro células o huecos, de $3,30 \times 3,60 \mathrm{~m}$ en planta, que se elevan desde la base hasta unos $2,40 \mathrm{~m}$ por debajo de la parte superior. Los tabiques o paredes que separan estos huecos tienen $0,60 \mathrm{~m}$ de espesor.

\section{encofrados deslizzantes}

La construcción de estas tres pilas principales se ha realizado utilizando encofrados exteriores e interiores, para formar las cuatro células, de 1,20 metros de altura, que se van elevando, a medida que se coloca el hormigón, mediante unos émbolos de apoyo que sobresalen y en los cuales se apoya el mecanismo de elevación compuesto de gatos hidráulicos. La velocidad discontinua con que se elevan los encofrados es de unos $2,5 \mathrm{~cm}$ por salto, produciéndose 12 saltos por hora, es decir, que el hormigón tiene una vejez de cuatro horas cuando la parte inferior del encofrado pasa sobre su nivel inicial.

La ejecución se continuó por tramos sucesivos en los que previamente se habían colocado las armaduras. La velocidad de marcha, en régimen normal, ha venido dada por el tiempo necesario para colocar las armaduras en posición horizontal. Las armaduras verticales son barras de $70 \mathrm{~mm}$ de diámetro, el cual disminuye en función de la altura hasta llegar a la parte superior, donde el diámetro de estos elementos es de 31 milímetros.

Antes de proceder al hormigonado se colocaron todas las armaduras, que se mantenían en posición apoyándolas en los costeros de los encofrados. Para ello se utilizaron entramados metálicos cuyos montantes quedaban embebidos en el hormigón, pero no así los elementos de arriostramiento, tanto horizontales como diagonales, ya que se iban recuperando a medida que el hormigonado avanzaba. Las barras verticales de las armaduras se iban soldando entre tramos, empleando el procedimiento de unir extremidad con extremidad, es decir, a tope. Para comprobar la calidad de estas soldaduras se siguieron procedimientos radioscópicos capaces de detectar cualquier imperfección.

Todos los elementos horizontales o diagonales de las armaduras se iban colocando a medida que avanzaba la ejecución.
Armaduras de las pilas principales.
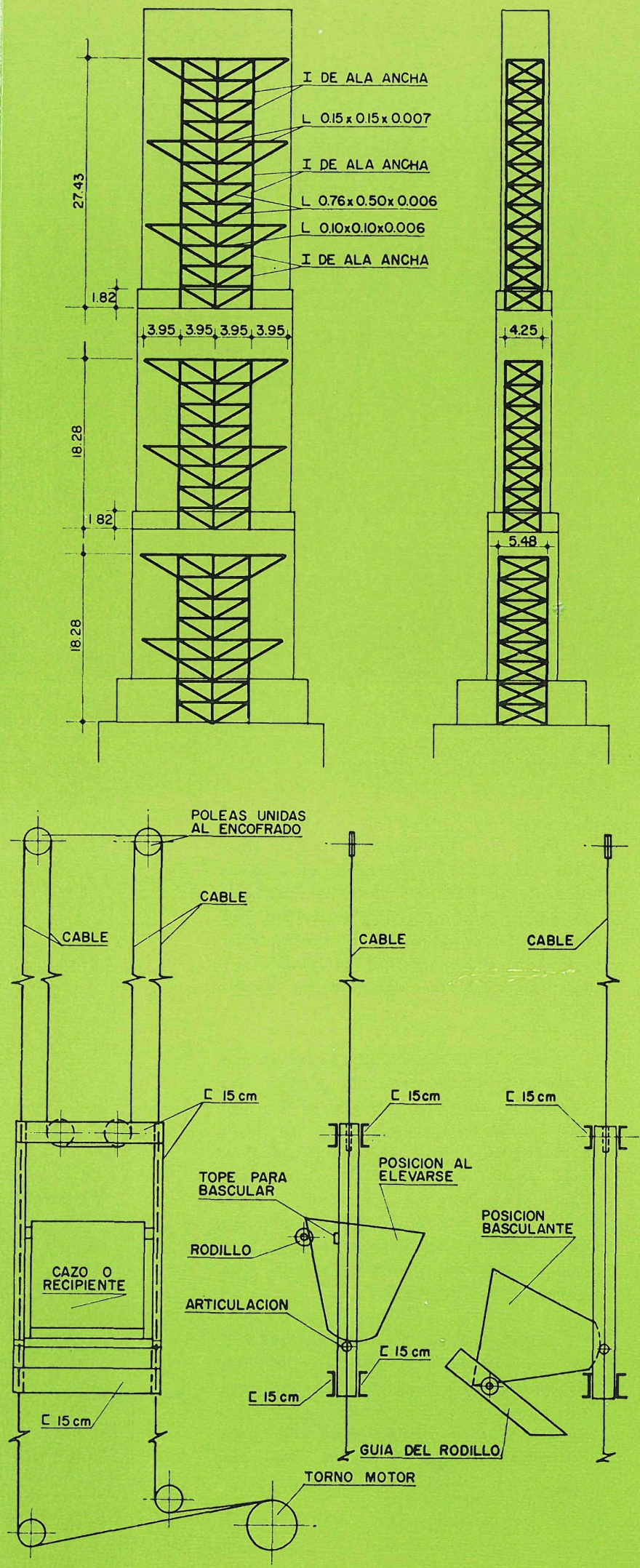

Bastidor y basculación del cazo. 


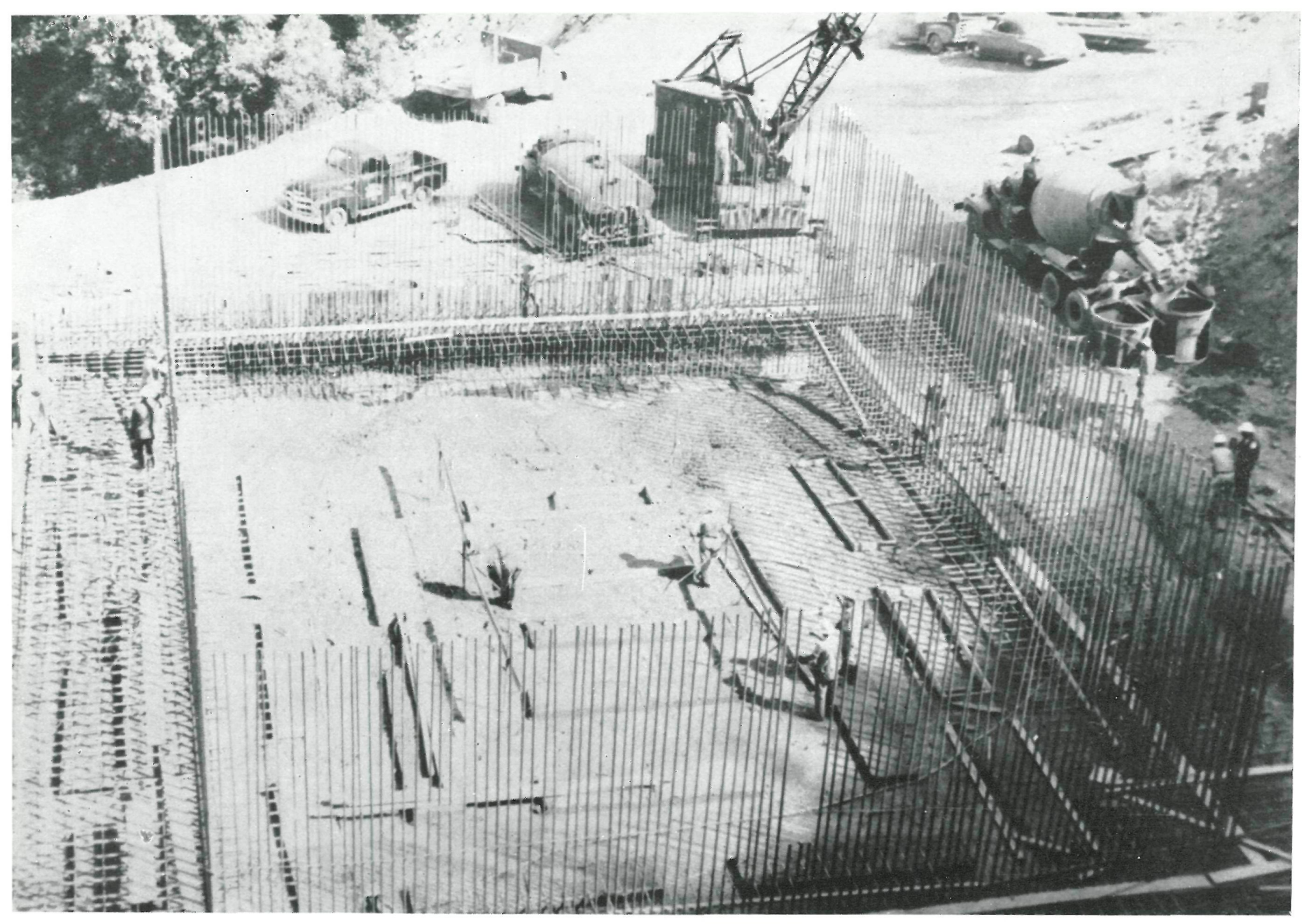

Para la elevación y manipula. ción de estos elementos se dispusieron dos plumas móviles que, accionadas por cables gobernados por tornos instalados en el suelo, trabajaban como grúas instaladas en los costeros de encofrados deslizantes.

Para el movimiento de estos últimos se emplearon 60 gatos, los cuales se movían, en elevación, sobre varillas verticales que se iban añadiendo, con juntas fileteadas, por trozos de $3,60 \mathrm{~m}$ de longitud. Cada una de estas varillas iba alojada en el interior de un tubo-lo que permitió su recuperación-para, finalmente, rellenarlo con una lechada de cemento.

Alrededor de los encofrados exteriores se instaló una plataforma de trabajo, y aprovechando los cuatro huecos de las células interiores se montó otra plataforma adicional complementaria, todo lo cual fue

Arranque de una pila.

Entramado de apoyo de armaduras.

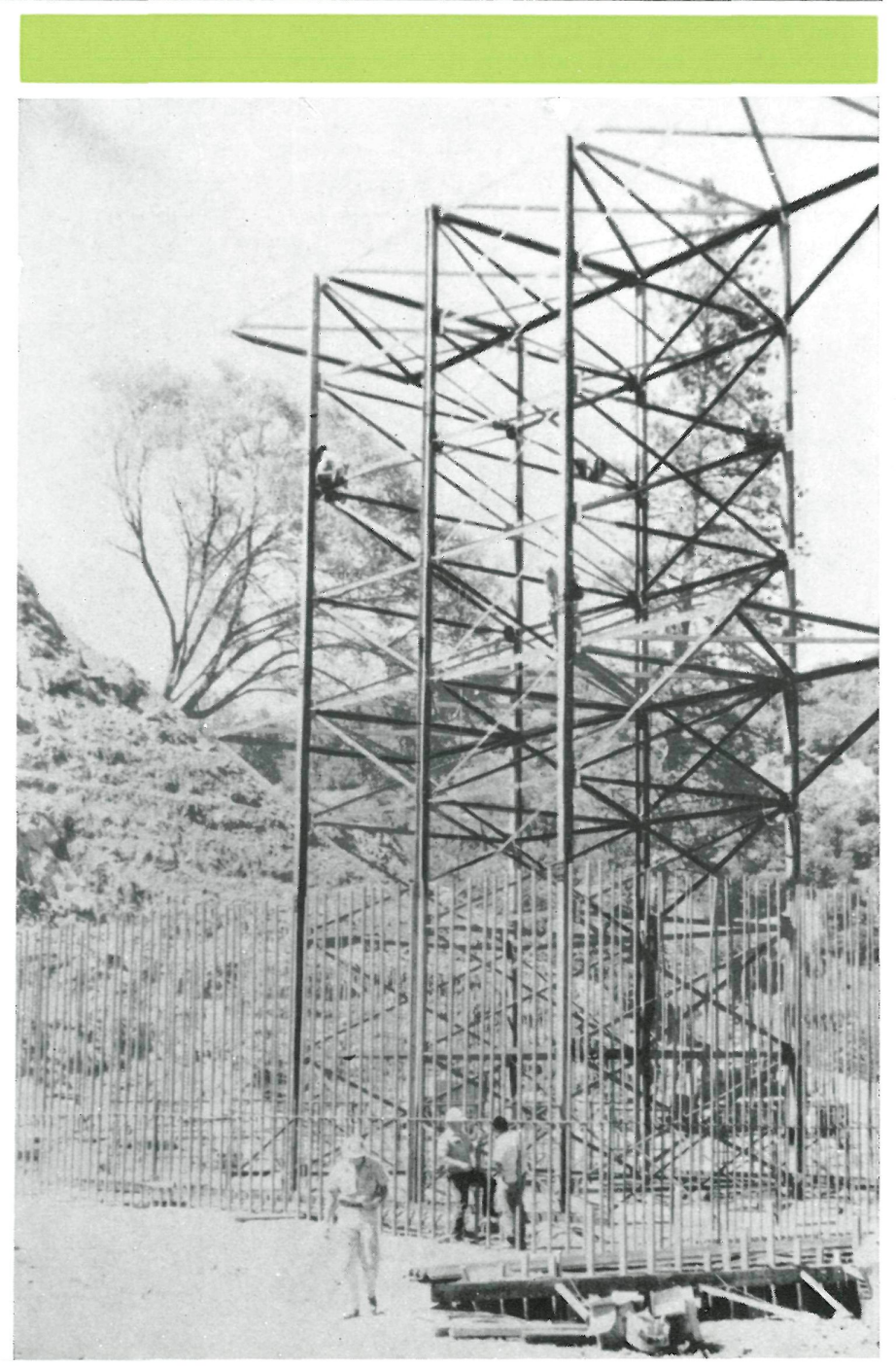




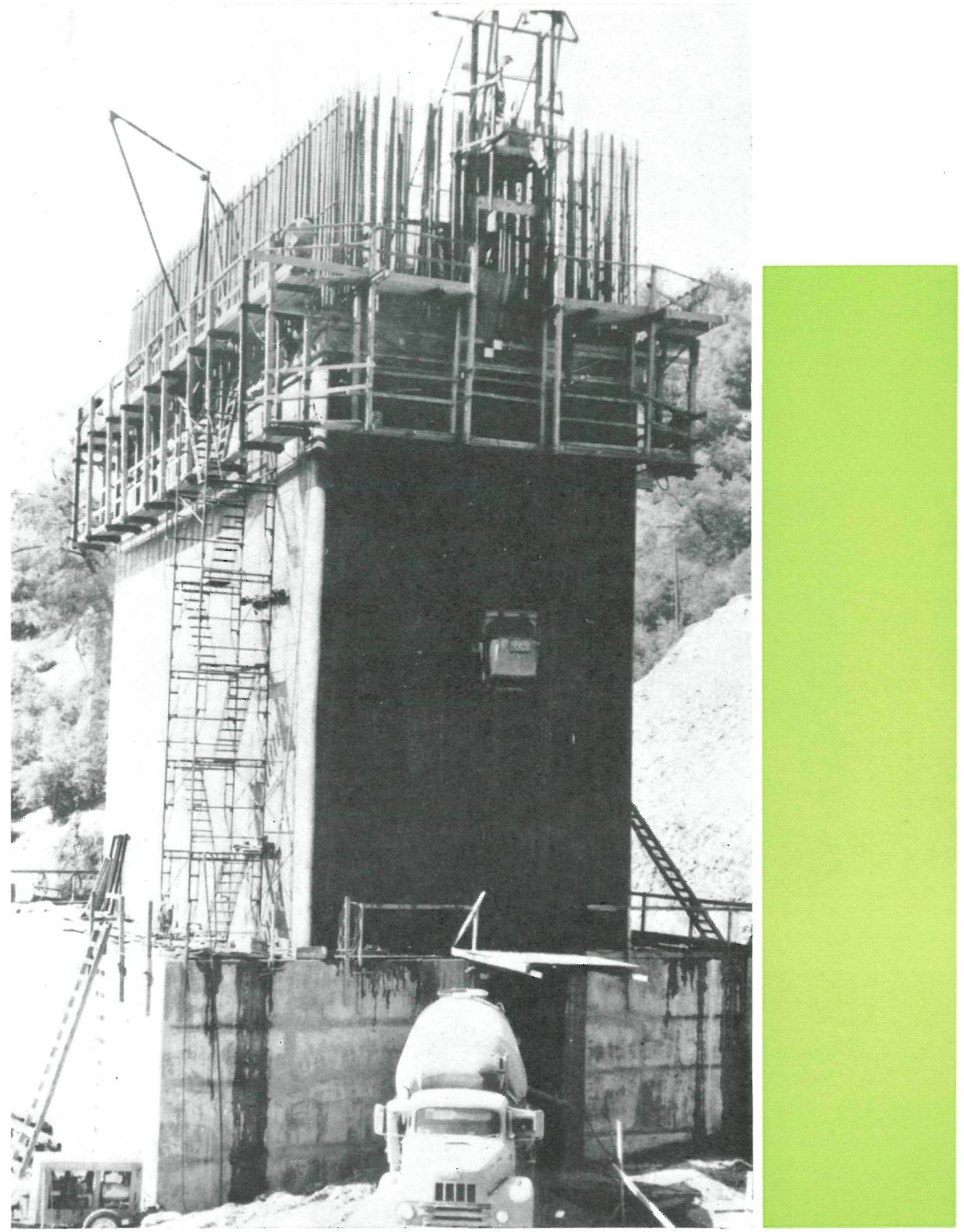

ncofrado deslizante sobre una pila

Pila terminada.

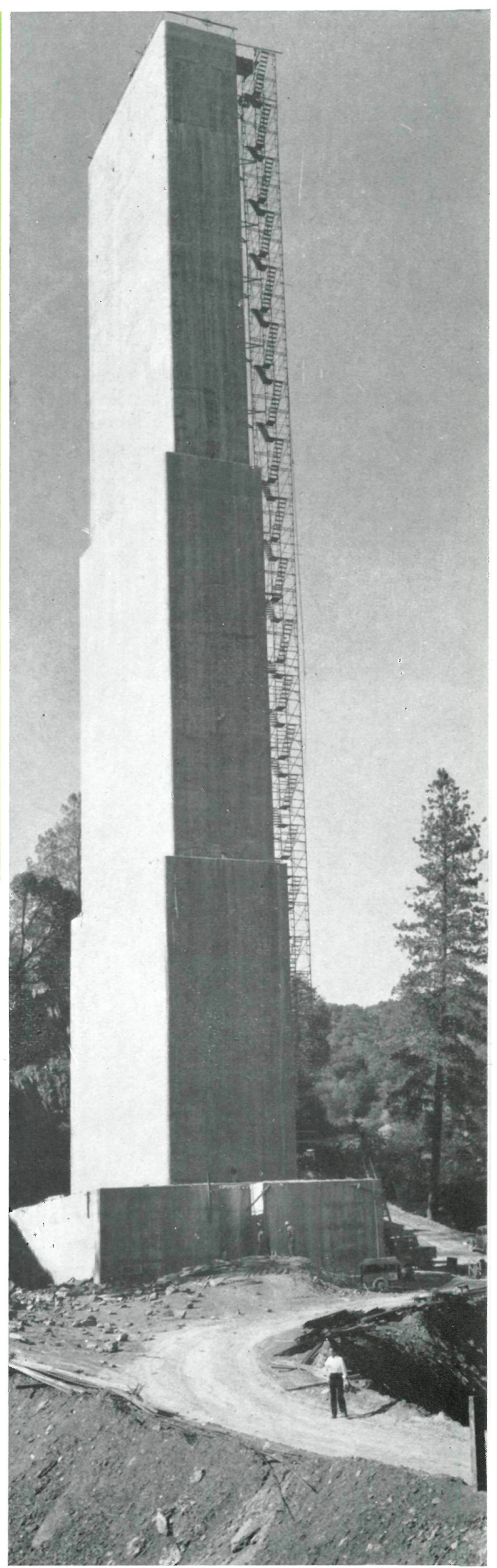

suficiente para permitir el acceso del personal a cualquier parte de la obra. Para las operaciones de acabado de los paramentos se colocó otra plataforma de trabajo en la parte inferior de los encofrados y otro tanto se hizo para las células interiores.

\section{tramsporte del |hormigón em elevación}

Para llevar el hormigón a las plataformas de trabajo se montaron dos torres, una en cada extremidad del encofrado, en el interior de las cuales se elevaba un recipiente que contenía el hormigón. Los recipientes con hormigón se vaciaban sobre otros móviles que lo distribuían en el tajo. El movimiento del bastidor que contenía el recipiente se maniobraba mediante unos tornos instalados en el suelo. Las comunicaciones entre conductores del torno y encargado del vaciado y distribución del hormigón en la plataforma de trabajo se mantuvieron por teléfono. 
Este procedimiento ha constituido, en cierto modo, una innovación, y en estos casos de gran altura, aventaja a los procedimientos tradicionales que emplean grúas provistas de plumas de gran longitud.

El curado se efectuó utilizando unos tubos para regar el hormigón, en los que se dejaron unos orificios, espaciados a $0,60 \mathrm{~m}$, que se instalaron en las plataformas para el acabado.

\section{los tableros}

La estructura metálica se empezó a montar a partir de la pila número 7 , después hasta la mitad del tramo 9-10 sobre el río. Luego se continuó el montaje a partir de la pila número 11, siguiendo hasta la clave del tramo 9-10. Para el montaje se emplearon los procedimientos cantilever, utilizando una grúa móvil que corría sobre la parte montada.

Dicha grúa situaba las partes de celosía, que debían añadirse a las anteriormente colocadas, sobre una mesilla, y de ésta, corriendo a lo largo de una vía sobre el tablero, se llevaban a la extremidad del tajo para su ulterior montaje.

Soporte metálico auxiliar.

Hormigonado de la losa superior.
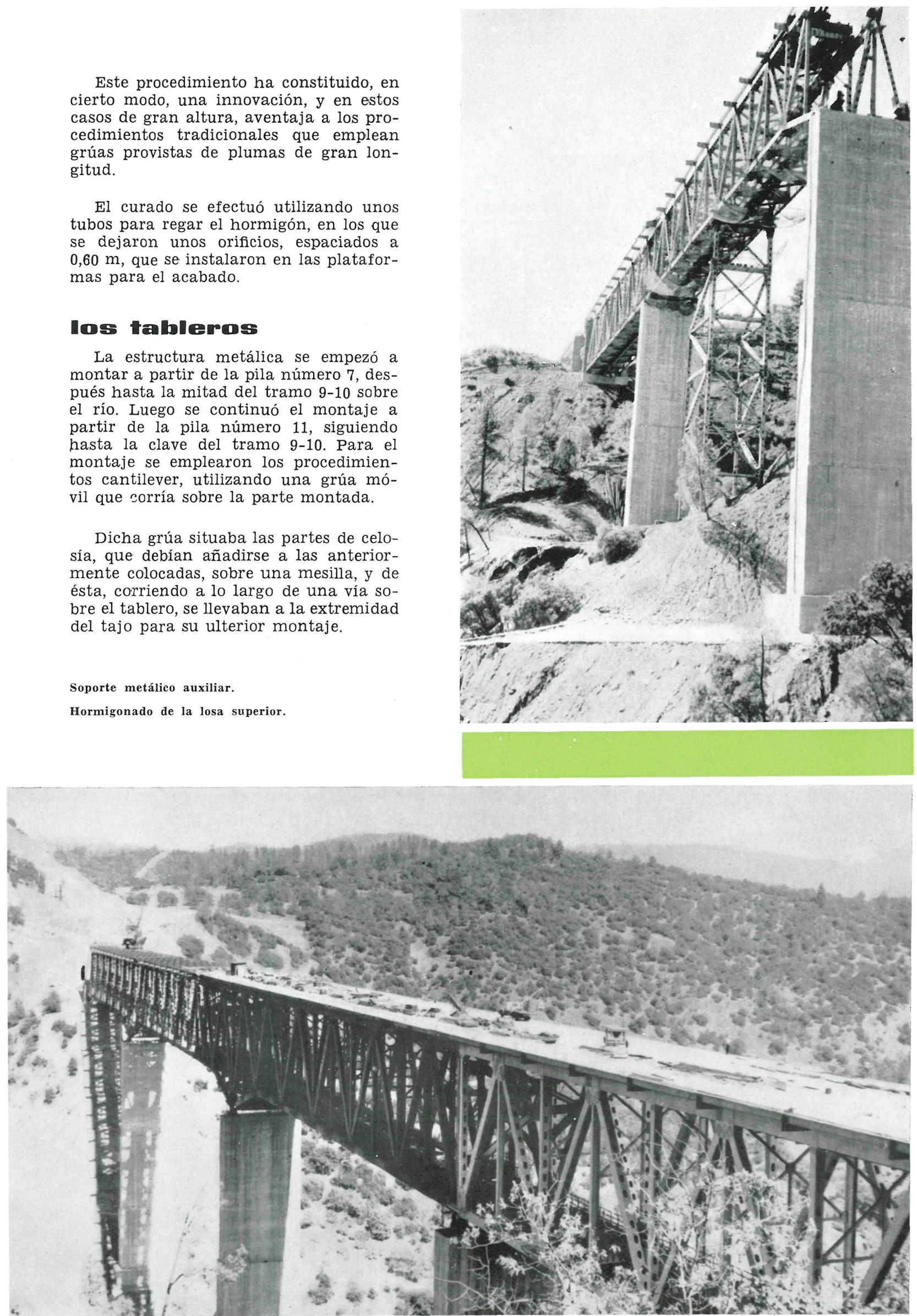


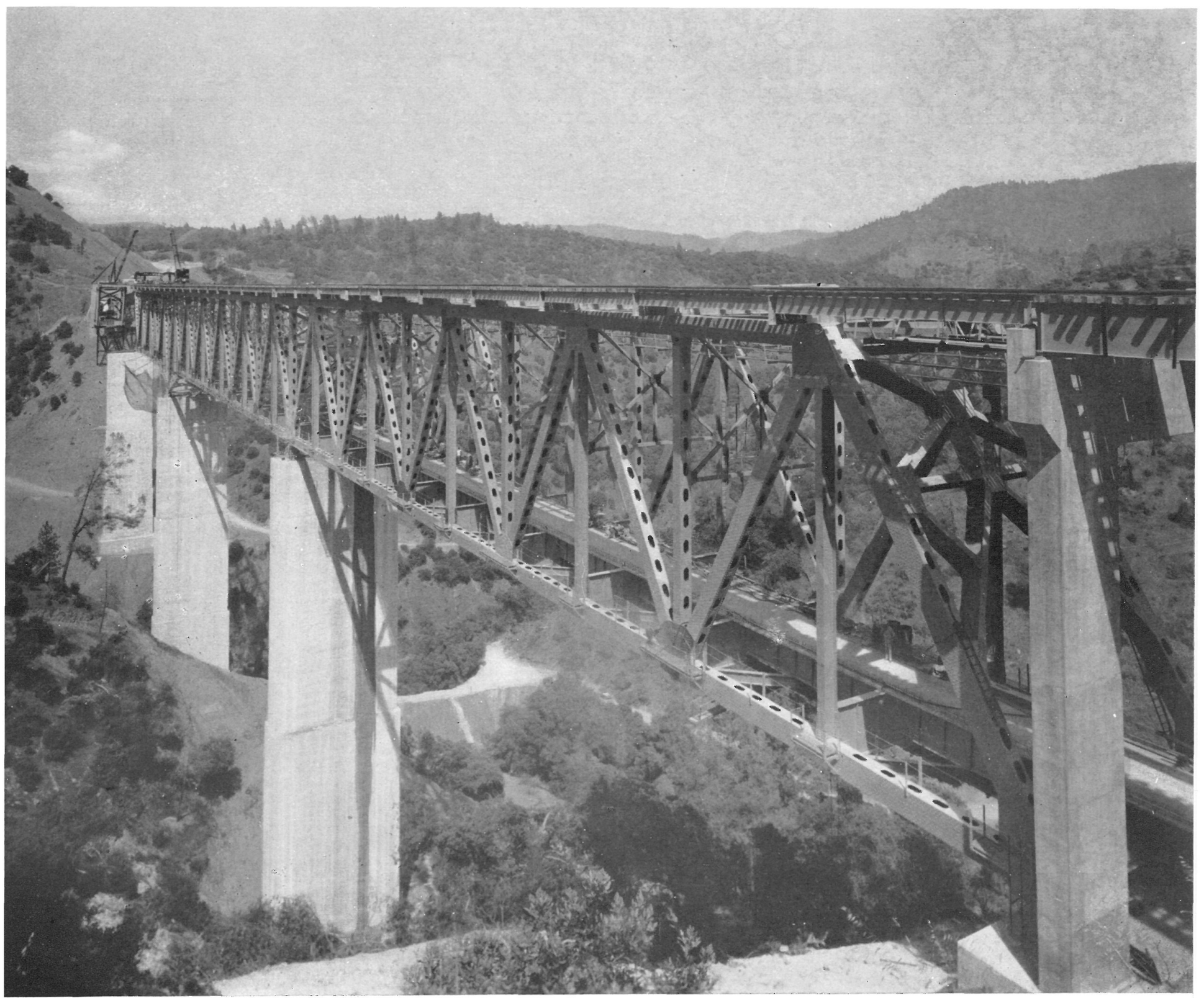

Entramado metálico de los dos tableros.

En estos tramos se utilizaron cinco soportes provisionales, que servían de apoyo parcial a la parte que quedaba en voladizo durante el montaje. El cierre de estos tramos se logró sin graves dificultades, salvo uno de ellos, que resultó, en la zona de cierre, con una longitud por exceso del orden de $9 \mathrm{mi}-$ límetros.

\section{elementos estructurales pesados}

Las partes de celosía de que se compone el tablero se prepararon en taller, empleando roblones en las uniones. El montaje en obra se ejecutó por medio de tornillos de alta resistencia. La totalidad de las celosías ha arrojado un peso de más de 11.000 t de acero laminado. El peso del mayor elemento montado fue de unas 56 toneladas.

Las losas, tanto del tablero superior para carreteras como las del inferior dedicadas a la plataforma para el balasto de la vía férrea, son de hormigón ligero.

El puente ha sido proyectado y dirigida la ejecución por el Departamento de Carreteras, de acuerdo con el State Department of Waters Rasources, y se ha publicado un resumen de la obra en la revista "California Highways and Public Works". 


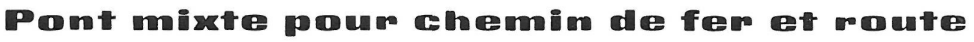

P. C. Harris, ingénieur.

Le nouveau pont, construit par la Département des Travaux Publics de Californie, appelé West Branch, franchit le cours profond d'une rivière. Il a un double but et comporte deux tabliers superposés: le supérieur supporte une route et l'autre une voie ferrée. La structure compte quatre sections principales appuyées sur des piles en béton armé, très elevées, dans lintérienr desquelles on été laissés quatre cellules ou creux de section rectangulaire. L'ensemble des deux tablers est composé d'une charpente métallique,
continue, montée suivant les procédés cantilever. Cette structure, à base d'éléments très lourds, d'environ $56 \mathrm{t}$ au maximum, a été montée en atelier par tronçons rivés et indépendants. Puis transportés au chantier, où ils furent unis entre eux pour former la structure définitive. Le montage a été exécuté à l'aide de boulons d'acier de haute résistance.

Comme les piles sont très hautes, leur fût présente des sections de dimensions différentes sur plan selon la cote. Le bétonnage de chacune de piles a été effectué au moyen de coffrages coulissants suffisamment hauts pour que le béton initiel coulé dans la partie Pour le bétonnage des piles, a été montée une tour auxiliaire qui servait d'appui au châssis portant la benne et son dispositif basculant. De cette benne le béton était versé dans d'autres récipients qui, à leur tour, le distribuaient dans les différentes parties de l'intérieur du coffrage. La manoeuvre de cet élévateur du béton, dont l'énergie était fournie par un tour installé au sol, était dirigée, par téléphone, entre l'opérateur du tour et le responsable de la distribution du béton sur la plate-forme supérieure de l'ouvrage. Pour le finissage des parements que le coffrage laissait en glissant vers le haut, on prépara une plate-forme auxiliaire de travail sur la partie inférieure du coffrage extérieur et la même chose pour les coffrages intérieurs correspondant aux quatre cellules ou creux intérieurs des niles.

\section{Combined Railwag and Roud Bridge}

P. C. Harris, engineer.

This new bridge, known as the West Branch, spans the course of a river, and has a double function and two superimposed decks. One of these carries the road, and the other lower one, the railway line. It has been constructed by the Dept. of Public Works of California.

The structure consists of four main spans resting on reinforced concrete piles, of great height. Four rectangular holes run down their centres. The decks consist of a continuous metal framework, made up of heavy elements, some of them weighing up to 56 tons each, was assembled in the workshop in separate sections. These were then
the whole structure. In the final assembly high tensile steel bolts were employed.

As the piles are very high, they are of varying cross section, according to the height. The concreting was done by means of sliding formwork, and this was sufficiently wide to ensure that the concrete was at least four hours old when the formwork
passed beyond its level.

To carry out these operations an auxiliary tower was built up. which supported the framowork carrying the concreting tub and its pouring mechanism. From this main tub the concrete was poured into others, which placed it into the various parts of the by telephone between the operator of the power plant and the person responsible for distributing the concrete on the top platform. To provide the finish of the surfaces left by the sliding formwork, another platform was installed, working at a level below the lower To provide the finish of the surfaces left by the sliding formwork, another platform was installed, working at a level below the lower
edge of the formwork, and a similar arrangement was adopted with respect to the inner formwork for the four hollows running edge of the form
inside the piles.

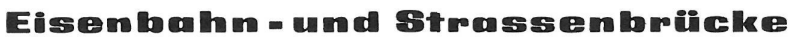

P. C. Harris, Ingenieur.

Die neue, West Branch genannte Brücke, welche das tiefe Bett eines Flusses überspannt, erfüllt einen doppelten Zweck; zwei übereinanderliegende Fahrbahnen dienen, die obere, für die Strasse, und die andere für eine Eisenbahnlinie. Sie wurde von der Abteilung der öfentlichen Arbeiten von California ausgeführt.

Das Gefüge besteht aus vier Hauptabschnitten, welche sich auf Stahlbeton-Pfeiler von grosser Höhe stützen, in deren Inneren man vier Zellen oder Kästen von rechteckigem Querschnitt gelassen hat. Die Gesamtheit der beiden Fahrbahnen wird von einem durchgehenden Stahlgefüge gebildet, das nach dem freitragenden Vorgehen, d. h. in Auskragung, aufgestellt wurde.

Das Fachwerk, auf Grund von maximal bis zu $56 \mathrm{t}$ schweren Elementen, wurde aus genieteten und unabhängigen Stücken in der Werkstatt zusammengestellt. Danach wurden sie zum Bau befördert, wo sie untereinander verbunden wurde
Gefüge zu bilden. Die Zusammenstellung erfolgte, indem man höchst widerständige Stahlschrauben benützte.

Da die Pfeiler von grosser Höhe sind, zeigt ihr Schaft im Grundriss, je nach der Höhe, Querschnitte verschiedener Grösse. Die Betonierung eines jeden der Pfeiler stellte man mit Gleitschalungen von genügender Höhe her, damit der Anfangsbeton im höheren Teile der Schalung vier Stunden alt wäre, wenn der untere Teil der Schalung an seinem Niveau voriberginge.

Für die Betonierung der Pfeiler stellte man einen Hilfskranz auf, der dem Rahmen, welcher den Topf und seine wippende Vorrichtung trug, als Stütze diente. Aus diesem Topfe oder Gefässe goss man den Beton in andere, welche ihn an den verschiedenen Teilen des Schalungsinneren liessen. Die Arbeit dieses Betonaufzuges, dessen Energie von einer auf dem Boden angelegten Drehbank geliefert wurde, hat man durch telefonische Mitteilung zwischen dem Drehbankführer und dem für die Verteilung des Betons auf der oberen Arbeitsplattform Beauftragten gelenkt. Für die Fertigung der Verblendungen, welche die Schalung beim Gleiten erhaben liess, wurde eine Arbeits-Hilfsplattform an dem
unteren Teile der äusseren Schalung hergerichtet, und dasselbe tat man für die entsprechenden Innenschalungen an den vier unteren Teile der äusseren Schalung herge
Zellen oder Kästen im Innern der Pfeiler. 